\title{
Majorana vortex-bound states in three-dimensional nodal noncentrosymmetric superconductors
}

\author{
Po-Yao Chang, ${ }^{1}$ Shunji Matsuura, ${ }^{2}$ Andreas P. Schnyder, ${ }^{3}$ and Shinsei Ryu ${ }^{1}$ \\ ${ }^{1}$ Department of Physics, University of Illinois at Urbana-Champaign, Urbana, Illinois 61801, USA \\ ${ }^{2}$ Department of Physics and Mathematics, McGill University, Montréal, Québec, Canada \\ ${ }^{3}$ Max-Planck-Institut für Festkörperforschung, Heisenbergstrasse 1, D-70569 Stuttgart, Germany
}

(Dated: November 4, 2018)

\begin{abstract}
Noncentrosymmetric superconductors (NCSs), characterized by antisymmetric spin-orbit coupling and a mixture of spin-singlet and spin-triplet pairing components, are promising candidate materials for topological superconductivity. An important hallmark of topological superconductors is the existence of protected zero-energy states at surfaces or in vortex cores. Here we investigate Majorana vortex-bound states in three-dimensional nodal and fully gapped NCSs by combining analytical solutions of Bogoliubov-de Gennes (BdG) equations in the continuum with exact diagonalization of BdG Hamiltonians. We show that depending on the crystal pointgroup symmetries and the topological properties of the bulk Bogoliubov-quasiparticle wave functions, different types of zero-energy Majorana modes can appear inside the vortex core. We find that for nodal NCSs with tetragonal point group $C_{4 v}$ the vortex states are dispersionless along the vortex line, forming one-dimensional Majorana flat bands, while for NCSs with $D_{4}$ point-group symmetry the vortex modes are helical Majorana states with a linear dispersion along the vortex line. NCSs with monoclinic point group $C_{2}$, on the other hand, do not exhibit any zero-energy vortex-bound states. We show that in the case of the $C_{4 v}\left(D_{4}\right)$ point group the stability of these Majorana zero modes is guaranteed by a combination of reflection ( $\pi$ rotation), time-reversal, and particle-hole symmetry. Considering continuous deformations of the quasiparticle spectrum in the presence of vortices, we show that the flat-band vortex-bound states of $C_{4 v}$ point-group NCSs can be adiabatically connected to the dispersionless vortex-bound states of time-reversal symmetric Weyl superconductors. Experimental implications of our results for thermal transport and tunneling measurements are discussed.
\end{abstract}

\section{Introduction}

Topological superconductors have in recent years become a subject of intense research due to their potential use for technical applications in device fabrication and quantum information 1-5. By the bulk-boundary correspondence, zeroenergy Majorana modes appear at the surface or inside the vortex core of topological superconductors. The experimental search for Majorana modes, which can be utilized as basic building blocks (i.e., half a qubit) for topological quantum computers, is the focus of a growing research effor ${ }^{6}$ ? These Majorana modes are robust against symmetry preserving impurity scattering processes and deformations of the superconducting order parameter. While topological superconductivity can be artificially engineered in heterostructures with an ordinary $s$-wave superconductor and, say, a semiconductor ${ }^{10}$ or a topological insulator ${ }^{13}$, it can also occur naturally in certain correlated materials with strong spin-orbit coupling (SOC).

One promising class of materials for topological superconductivity are the noncentrosymmetric superconductors $(\mathrm{NCSs}){ }^{14}$. In these systems, the absence of inversion symmetry together with strong SOC and electronic correlations can give rise to unconventional pairing states with topologically nontrivial characteristics 15,24 . For example, in $\mathrm{CePt}_{3} \mathrm{Si}^{25}-27$, macroscopic as well as microscopic measurements indicate an unconventional superconducting state with a mixture of spinsinglet and spin-triplet pairing components and line nodes in the superconducting gap. Experimental evidence for unconventional pairing symmetries has also been reported for $\mathrm{CeIrSi}_{3}$, $28, \mathrm{CeRhSi}_{3}{ }^{29}, \mathrm{Y}_{2} \mathrm{C}_{3} \sqrt{30}, \mathrm{Li}_{2} \mathrm{Pt}_{3} \mathrm{~B}^{31}, 33$, and $\mathrm{BiPd}^{34}$. Both fully gapped and nodal NCSs with sizable spin-triplet pairing components exhibit nontrivial topological properties, which manifest themselves in terms of different types of zero- energy surface states. In fully gapped NCSs the surface states are dispersing helical Majorana modes, whereas nodal NCSs exhibit flat-band surface states $\frac{16 \mid 19}{222] 35}$, and depending on the crystallographic point group, may also support helical Majorana modes or arc surface states 23 . Experimentally, it is possible to distinguish among different types of surface states using Fourier-transform scanning tunneling spectroscopy 36 or surface transport measurements 3738 .

Most of the candidate materials for noncentrosymmetric topological superconductivity are strong type-II superconductors, with Ginzburg-Landau parameters $\kappa$ of the order of $\sim 100^{14}$. Hence, zero-energy Majorana modes may emerge inside magnetic vortices of these superconducting compounds 2439 41. In this paper, we examine vortex-bound states of three-dimensional (3D) NCSs and study how their appearance is related to the crystal point-group symmetries of the superconductor and the nontrivial topological properties of the bulk Bogoliubov-quasiparticle wave functions. Using both numerical and analytical methods, we compute the vortex-bound state spectra of $(s+p)$-wave NCSs with three different point-group symmetries: the two tetragonal pointgroups $D_{4}$ and $C_{4 v}$, as well as the monoclinic point-group $\mathrm{C}_{2} \stackrel{42}{\text {. }}$

One of our primary findings is that $D_{4}$ point-group NCSs support gapless helical Majorana states inside vortex cores. These subgap states disperse linearly along the vortex line, and are akin to one-dimensional helical Majorana modes that exist at the edge of fully gapped topological NCSs in two dimensions. Remarkably, these vortex-bound states appear both in the fully gapped topological phase and in the nodal phase that separates the fully gapped trivial phase from the topological one [see Figs. 1 (a) and 2]. While these helical Majorana vortex states exist in an extended region of the phase diagram 
of Fig. 1.a), they are unstable against perturbations that break the $D_{4}$ point-group symmetry of the superconductor $(\mathrm{SC})^{43}$. For NCSs with tetragonal point-group symmetry $C_{4 v}$, on the other hand, we find that there are zero-energy vortex-bound states which are dispersionless along the vortex line, forming a one-dimensional Majorana flat band (Fig. 3). In contrast, $\mathrm{C}_{2}$ point-group NCSs do not exhibit any zero-energy vortexbound states neither in the fully gapped nor in the nodal phase (Fig. 4). We find that the Majorana vortex-bound states of the $C_{4 v}\left(D_{4}\right)$ point-group NCS are protected by a combination of reflection ( $\pi$ rotation), time-reversal, and particle-hole symmetry.

Interestingly, the existence of these vortex-bound states in nodal NCSs correlates to some degree with the appearance of extra surface states, that appear in addition to the flat-band surface states. That is, for nodal NCSs with $D_{4}$ point-group symmetry the helical vortex-bound states always appear together with helical Majorana cones on the surface which are protected by a $\mathbb{Z}_{2}$ topological number ${ }^{1923}$. (In the following, we refer to these Majorana cone surface states as the " $\mathbb{Z}_{2}$ surface states.") On the other hand, for nodal $C_{4 v}$ point-group NCSs the existence of flat-band vortex states is correlated with the appearance of helical arc states on the surface ${ }^{23}$, see Table I. These arc surface states are superconducting analogues of the Fermi arcs that exist on the surface of Weyl semimetals 44 46. Using translation symmetry in the vortex direction, we fix the momentum along the vortex line and consider adiabatic deformations of the quasiparticle spectrum that do not close the bulk energy gap for this fixed momentum. By use of this procedure, we find that the vortex-bound states (extra surface states) of $D_{4}$ and $C_{4 v}$ point-group NCSs are adiabatically connected to the vortex-bound states (surface states) of fully gapped topological SCs and time-reversal symmetric Weyl SCs, respectively. Conversely, finite-energy vortexbound states of nodal NCSs with $C_{2}$ point-group symmetry can be related to finite-energy vortex-bound states of fully gapped trivial SCs (cf. Table I).

\section{Model Hamiltonian and symmetries}

To study the appearance of vortex-bound states in nodal NCSs, we consider a generic single-band Bogoliubov-de Gennes (BdG) Hamiltonian $H=\sum_{\mathbf{k} \in \mathrm{BZ}} \Psi_{\mathbf{k}}^{\dagger} \mathcal{H}(\mathbf{k}) \Psi_{\mathbf{k}}$, with

$$
\mathcal{H}(\mathbf{k})=\left(\begin{array}{cc}
h(\mathbf{k}) & \Delta(\mathbf{k}) \\
\Delta^{\dagger}(\mathbf{k}) & -h^{T}(-\mathbf{k})
\end{array}\right)
$$

and the Nambu spinor $\Psi_{\mathbf{k}}=\left(c_{\mathbf{k} \uparrow}, c_{\mathbf{k} \downarrow}, c_{-\mathbf{k} \uparrow}^{\dagger}, c_{-\mathbf{k} \downarrow}^{\dagger}\right)^{\mathrm{T}}$, where $c_{\mathbf{k} \sigma}\left(c_{\mathbf{k} \sigma}^{\dagger}\right)$ denotes the electron annihilation (creation) operator with momentum $\mathbf{k}$ and $\operatorname{spin} \sigma=\uparrow, \downarrow$. The normal-state Hamiltonian $h(\mathbf{k})=\varepsilon(\mathbf{k}) \mathbb{I}_{2 \times 2}+\alpha \mathbf{l}(\mathbf{k}) \cdot \boldsymbol{\sigma}$ describes electrons on a cubic lattice with nearest-neighbor hopping $t$, chemical potential $\mu$, spin-independent dispersion $\varepsilon(\mathbf{k})=t\left(\cos k_{x}+\right.$ $\left.\cos k_{y}+\cos k_{z}\right)-\mu$, and Rashba-type SOC $\alpha \mathbf{l}(\mathbf{k}) \cdot \boldsymbol{\sigma}$ with strength $\alpha$. Here, $\boldsymbol{\sigma}=\left(\sigma_{1}, \sigma_{2}, \sigma_{3}\right)$ is the vector of Pauli matrices. Due to the absence of inversion symmetry, the superconducting gap $\Delta(\mathbf{k})$ contains in general an admixture of even-
TABLE I. Depending on the crystal point-group symmetries (first column), nodal NCSs can exhibit different types of zero-energy vortex-bound states (second column). As indicated in the third column, the appearance of these different vortex states correlates with the existence of extra surface states besides the flat-band states. The helical vortex states and the $\mathbb{Z}_{2}$ surface states of nodal $D_{4}$ NCSs can be adiabatically connected to the vortex-bound and surface states of fully gapped topological NCSs. Similarly, the flat-band vortex states and arc surface states of $C_{4 v}$ NCSs are related to the vortex-bound and surface states of time-reversal symmetric Weyl SCs.

\begin{tabular}{|l|c|c|c|}
\hline & Vortex states & Extra surface states & Adiabatic deformation \\
\hline$D_{4}$ & helical states & $\mathbb{Z}_{2}$ Majorana cone & fully gapped top. SC \\
\hline$C_{4 v}$ & flat bands & helical arc states & Weyl SC with TRS \\
\hline$C_{2}$ a & none & none & gapped trivial SC \\
\hline
\end{tabular}

${ }^{\mathrm{a}}$ for phase IV in Fig. 1(c).

parity spin-singlet and odd-parity spin-triplet pairing components, $\Delta(\mathbf{k})=\left(\Delta_{s} \mathbb{I}_{2 \times 2}+\Delta_{t} \mathbf{d}(\mathbf{k}) \cdot \boldsymbol{\sigma}\right)\left(i \sigma_{2}\right)$, where $\Delta_{s}$ and $\Delta_{t}$ represent the spin-singlet and spin-triplet pairing amplitudes, respectively. For the spin-triplet pairing term we assume that the vector $\mathbf{d}(\mathbf{k})$ is oriented parallel to the polarization vector $\mathbf{l}(\mathbf{k})$ of the $\mathrm{SOC}^{47}$. To simplify matters we will set $\left(t, \alpha, \Delta_{t}\right)=(-1,1,1)$ in our numerical calculations and study the vortex-bound states as a function of $\Delta_{s}, \mu$, and different types of SOC potentials. We have checked that different values of $\left(t, \alpha, \Delta_{t}\right)$ do not qualitatively change our results. With $\varepsilon(\mathbf{k})=\varepsilon(-\mathbf{k})$ and $\mathbf{l}(\mathbf{k})=-\mathbf{l}(-\mathbf{k})$, Hamiltonian (1) is invariant under both time-reversal symmetry (TRS) and particle-hole symmetry (PHS),

$$
U_{T}^{-1} \mathcal{H}(\mathbf{k}) U_{T}=\mathcal{H}^{*}(-\mathbf{k})
$$

and

$$
U_{P}^{-1} \mathcal{H}(\mathbf{k}) U_{P}=-\mathcal{H}^{*}(-\mathbf{k})
$$

where $U_{T}=\mathbb{I}_{2 \times 2} \otimes i \sigma_{2}$ and $U_{P}=\sigma_{1} \otimes \mathbb{I}_{2 \times 2}$, respectively. Hence, since $U_{T} U_{T}^{*}=-\mathbb{I}_{4 \times 4}$ and $U_{P} U_{P}^{*}=\mathbb{I}_{4 \times 4}, \mathcal{H}(\mathbf{k})$ belongs to symmetry class DIII.

The specific form of the spin-orbit coupling vector $\mathbf{l}(k)$ is constrained by the lattice symmetries of the superconductor ${ }^{48}$. In the following we consider NCSs with three different crystal point-group symmetries: the tetragonal point groups $D_{4}$ and $C_{4 v}$, as well as the monoclinic point group $C_{2}$. Within a tightbinding expansion, we obtain for the crystal point group $D_{4}$ to lowest order

$$
\mathbf{l}(\mathbf{k})=\left(a_{1} \sin k_{x}, a_{1} \sin k_{y}, a_{2} \sin k_{z}\right) .
$$

For the tetragonal point group $C_{4 v}$, which is relevant for $\mathrm{CePt}_{3} \mathrm{Si}, \mathrm{CeRhSi}_{3}$, and $\mathrm{CeIrSi}_{3}$, the vector $\mathbf{l}(\mathbf{k})$ takes the form

$$
\mathbf{l}(\mathbf{k})=a_{1}\left(\sin k_{y},-\sin k_{x}, 0\right) .
$$

The lowest order terms compatible with $C_{2}$ point-group symmetry (represented by BiPd) are given by

$\mathbf{l}(\mathbf{k})=\left(a_{1} \sin k_{x}+a_{5} \sin k_{y}, a_{2} \sin k_{y}+a_{4} \sin k_{x}, a_{3} \sin k_{z}\right)$. 
$D_{4}$ and $C_{4 v}$ NCSs exhibit, besides the global symmetries TRS and PHS, also rotation and reflection symmetries. Two of these crystalline symmetries play an important role for the protection of zero-energy vortex-bound states. Let us discuss these in more detail. We find that the $D_{4}$ NCS is invariant under $\pi$ rotation along the $x$ axis, which acts on the Hamiltonian (1) as

$$
U_{\mathrm{R}_{\pi}}^{\dagger} \mathcal{H}\left(\mathrm{R}_{\pi} \mathbf{k}\right) U_{\mathrm{R}_{\pi}}=\mathcal{H}(\mathbf{k}),
$$

where $U_{\mathrm{R}_{\pi}}=\operatorname{diag}\left(u_{\mathrm{R}_{\pi}}, u_{\mathrm{R}_{\pi}}^{*}\right)$ and $u_{\mathrm{R}_{\pi}}$ is the spinor representation of the rotation $\mathrm{R}_{\pi}=\operatorname{diag}(1,-1,-1)$, i.e., $u_{\mathrm{R}_{\pi}}=$ $\exp \left[-i(\pi / 2) \sigma_{1}\right]=-i \sigma_{1}$. The $C_{4 v}$ point-group NCS, on the other hand, satisfies the reflection symmetry

$$
U_{\mathrm{R}_{y}}^{\dagger} \mathcal{H}\left(\mathrm{R}_{y} \mathbf{k}\right) U_{\mathrm{R}_{y}}=\mathcal{H}(\mathbf{k}),
$$

with $U_{\mathrm{R}_{y}}^{\dagger}=\operatorname{diag}\left(u_{\mathrm{R}_{y}}, u_{\mathrm{R}_{y}}^{*}\right)$ and $u_{\mathrm{R}_{y}}=i \sigma_{2}$ the spinor representation of the reflection operator $\mathrm{R}_{y}=\operatorname{diag}(1,-1,1)$.

For a pair of vortex-antivortex lines oriented along the $z$ axis, the spin-singlet and spin-triplet order parameters are modified as

$$
\Delta_{s, t}(x, y)=\Delta_{s, t} e^{i \phi(x, y)},
$$

where the phase angle $\phi(x, y)$ is given by $\phi(x, y)=$ $\tan ^{-1}\left[2 a b y /\left(x^{2}+(b y)^{2}-a^{2}\right)\right]$. This describes a vortex and antivortex line with winding number \pm 1 located at $(a, 0)$ and $(-a, 0)$, respectively. The anisotropy of the vortex line is controlled by the parameter $b$. In order to compute the vortexbound states we set $(a, b)=(8,2)$ and diagonalize the BdG Hamiltonian (1) on a $50 \times 50 \times 60$ cubic lattice with periodic boundary conditions (PBCs) in all three directions. To simplify matters, we do not take into account the Zeeman effect. Most NCS topological superconductor candidate materials are extreme type-II superconductor ${ }^{14}$ with a lower critical field $H_{c 1}$ of the order of $1 \mathrm{mT}$, corresponding to an energy scale an order of magnitude smaller than the gap energy. For these systems, it is expected that the Zeeman effect can be neglected for a magnetic field that is not much larger than $H_{c 1}$.

The Hamiltonian in the presence of a pair of vortexantivortex lines along the $z$ axis breaks TRS as well as the crystal symmetries (4) and (5), but remains invariant under a combination of TRS with crystal symmetries. That is, the $D_{4}$ point-group NCS with $k_{z}=0$ satisfies the following symmetry

$$
\left[\mathcal{U}_{\mathrm{R}_{\pi}} \mathcal{U}_{T}\right]^{-1} \widetilde{\mathcal{H}}\left(x, x^{\prime}, y, y^{\prime}\right)\left[\mathcal{U}_{\mathrm{R}_{\pi}} \mathcal{U}_{T}\right]=\widetilde{\mathcal{H}}^{*}\left(x, x^{\prime}, y, y^{\prime}\right),
$$

where $\widetilde{\mathcal{H}}\left(x, x^{\prime}, y, y^{\prime}\right)$ denotes the Fourier transform of $\mathcal{H}\left(k_{x}, k_{y}, k_{z}=0\right), \mathcal{U}_{\mathrm{R}_{\pi}}=\delta_{x, x} \delta_{y,-y} U_{\mathrm{R}_{\pi}}$ is the real-space $\pi$-rotation operator, and $\mathcal{U}_{T}=\delta_{x, x} \delta_{y, y} \otimes U_{T}$ represents the time-reversal operator in position space. Similarly, for the $C_{4 v}$ NCS in the presence of vortices, we find the following symmetry

$$
\left[\mathcal{U}_{\mathrm{R}_{y}} \mathcal{U}_{T}\right]^{-1} \widetilde{\mathcal{H}}\left(x, x^{\prime}, y, y^{\prime}, k_{z}\right)\left[\mathcal{U}_{\mathrm{R}_{y}} \mathcal{U}_{T}\right]=\widetilde{\mathcal{H}}^{*}\left(x, x^{\prime}, y, y^{\prime}, k_{z}\right),
$$

for all $k_{z}$, where $\mathcal{U}_{\mathrm{R}_{y}}=\delta_{x, x} \delta_{y,-y} \otimes U_{\mathrm{R}_{y}}$ denotes the reflection operator in real space. Here, the matrix $\delta_{x, x} \delta_{y,-y}$ acts on the real-space basis, while $U_{\mathrm{R}_{y}}$ acts on the Nambu basis. We note that the $C_{2}$ NCSs does not possess any symmetry of the form of Eqs. (7). In Sec. IV it is shown that symmetries (7) together with PHS lead to the protection of zero-energy vortexbound states.

\section{Phase diagram and topological invariants}

The phase diagram of Hamiltonian (1) in the absence of vortices is shown in Figs. 1 (a) -1 (c) as a function of spin-singlet pairing amplitude $\Delta_{s}$ and chemical potential $\mu$. Two fully gapped phases with trivial and nontrivial topology (phases I and II in Fig. 1) are separated by a nodal superconducting phase (phases III and IV in Fig. $1{ }^{49}$. Interestingly, for the $C_{2}$ point-group NCS we find that there are two distinct gapless phases with a Lifshitz transition in between, at which the nodal rings touch each other and reconnect in a different manner [see Figs. 1(d) and 4(a)-4(d)]. The topological properties of the fully gapped phases I and II in Fig. 1 are characterized by the $3 \mathrm{D}$ winding number $\nu_{3},{ }^{19]}$ which is defined as

$$
\nu_{3}=\int_{\mathrm{BZ}} \frac{d^{3} k}{24 \pi^{2}} \epsilon^{\mu \nu \rho} \operatorname{Tr}\left[\left(q^{-1} \partial_{k_{\mu}} q\right)\left(q^{-1} \partial_{k_{\nu}} q\right)\left(q^{-1} \partial_{k_{\rho}} q\right)\right],
$$

where $q$ is the off-diagonal block of the spectral projector, see Appendix A We find that phase I is topologically nontrivial with $\nu_{3}=-1$, while phase II is trivial with $\nu_{3}=0$. Note that this 3D winding number $\nu_{3}$ is only well defined for fully gapped phases. The topological characteristics of the nodal phases III and IV, however, can be described by the onedimensional winding number ${ }^{191222}$

$$
\nu_{1}=\frac{i}{2 \pi} \oint_{\mathcal{L}} d k_{\mu} \operatorname{Tr}\left[q^{-1} \partial_{k_{\mu}} q\right]
$$

where $\mathcal{L}$ is a closed path that interlinks with a line node. In both nodal phases III and IV, the winding number $\nu_{1}$ evaluates to \pm 1 for each nodal ring. To characterize the nodal phases it is also possible to define a one- or two-dimensional $\mathbb{Z}_{2}$ topological invariant ${ }^{19 \mid 22]}$

$$
W_{\mathcal{A}}=\prod_{\mathbf{K}} \frac{\operatorname{Pf}\left[q^{T}(\mathbf{K})\right]}{\sqrt{\operatorname{det}[q(\mathbf{K})]}},
$$

where $\mathcal{A}$ is a time-reversal invariant line or plane embedded in the 3D Billouin zone (BZ) and $\mathbf{K}$ denotes the two (four) time-reversal invariant momenta on the line (plane) $\mathcal{A}$.

\section{Vortex-bound states and surface states}

In this section we discuss the surface states and vortexbound states of nodal NCS with the thee different point-group symmetries $D_{4}, C_{4 v}$, and $C_{2}$. 
(a)

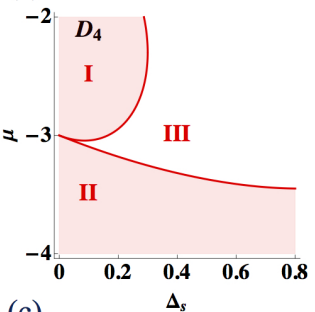

(c)

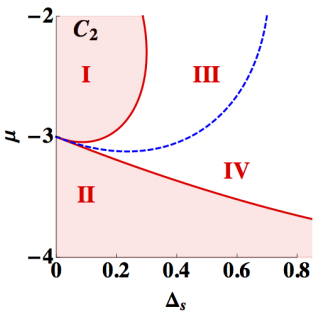

$\Delta_{s}$

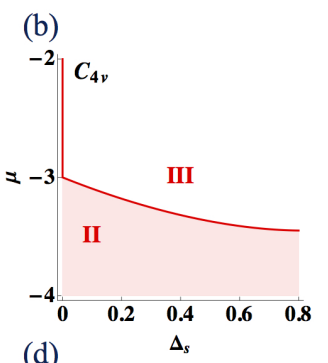

(d)

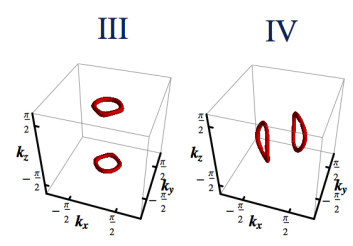

FIG. 1. (Color online). Phase diagram of Hamiltonian (1) as a function of spin-singlet pairing amplitude $\Delta_{s}$ and chemical potential $\mu$ for the point group (a) $D_{4}$ with $\left(a_{1}, a_{2}\right)=(1.0,0.3)$, (b) $C_{4 v}$ with $a_{1}=1.0$, and (c) $C_{2}$ with $\left(a_{1}=a_{2}, a_{3}, a_{4}=a_{5}\right)=(1.0,0.3,0.5)$. The fully gapped phases (red shaded regions) are characterized by the winding number $\nu_{3}$, where $\nu_{3}=-1$ (phase I) corresponds to the topological phase, while $\nu_{3}=0$ (phase II) is the trivial phase. The blue dashed line in panel (c) represents the phase boundary between the two distinct nodal structures III and IV shown in panel (d).

\section{A. $D_{4}$ point-group NCSs}

We start by considering a nodal NCS with $D_{4}$ point-group symmetry in phase III of Fig. 1.a). In this region of parameter space the bulk Bogoliubov quasiparticle spectrum exhibits two topologically stable nodal rings, which are centered about the (001) axis [Figs. 2(a) and 2,b)]. The onedimensional winding number $\nu_{1}$ (topological charge) of these two nodal rings is $\nu_{1}= \pm 1$, which by the bulk-boundary correspondence results in the appearance of flat-band surface states $19\left[23\right.$. In addition to the surface flat bands, nodal $D_{4}$ NCSs exhibit $\mathbb{Z}_{2}$ Majorana surface states. This is shown in Figs. 2.c) and 2(d) for the (100) surface, where a helical Majorana cone appears at $\left(k_{y}, k_{z}\right)=(0,0)$ of the surface BZ. As shown in Ref. 19, this Majorana surface state is protected by the one-dimensional $\mathbb{Z}_{2}$ topological invariant (10) with $\mathcal{A}$ a time-reversal invariant line. Choosing $\mathcal{A}$ to be oriented along the $k_{x}$ axis with $\left(k_{y}, k_{z}\right)$ held fixed, we find that $W_{\mathcal{A}}=-1$ at $\left(k_{y}, k_{z}\right)=(0,0)$, which signals the appearance of a zeroenergy helical Majorana state. At the other three time-reversal invariant momenta of the surface BZ there are no surface states, in agreement with $W_{\mathcal{A}}=+1$ for theses surface momenta.

$D_{4}$ point-group NCSs support zero-energy helical Majorana states not only on the surface but also inside vortex cores. This is illustrated in Fig. 2(e), which shows the energy spectrum in the presence of a pair of vortex and antivortex lines oriented along the $z$ axis. At energies smaller than the bulk gap there appear vortex-bound states which disperse linearly

(a)

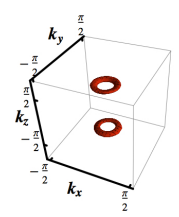

(d)

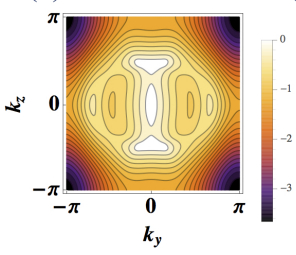

(b)

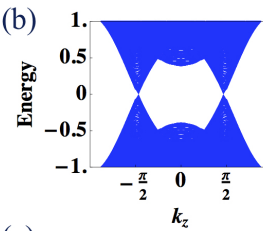

(e)

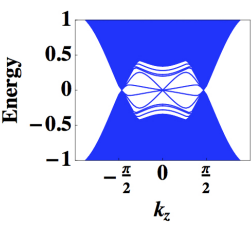

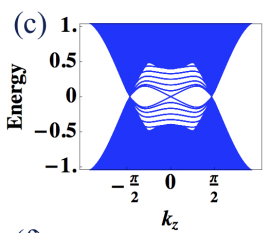

(f)

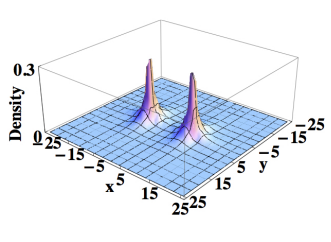

FIG. 2. (Color online). Zero-energy vortex-bound states and surface states in a $\mathrm{D}_{4}$ point-group NCS with $\left(a_{1}, a_{2}\right)=(1.0,0.3)$, $\mu=-2.5$, and $\Delta_{s}=0.5$. This parameter choice corresponds to phase III in Fig. 1 (a). Energies are measured in the unit of hopping. (a) Bulk nodal structure. (b) and (c): Energy spectrum in the absence of vortices as a function of $k_{z}$ with (b) PBCs in all three directions and (c) OBCs along the $x$ axis but PBCs in the other two directions. (d) Energy dispersion of the highest negative-energy state of the $D_{4}$ NCS in a (100) slab geometry. The color scale is such that white represents zero energy. (e) Energy spectrum as a function of $k_{z}$ in the presence of a vortex-antivortex pair oriented along the $z$ axis. The subgap states are localized at the vortex cores. (f) Probability distribution of the zero-energy vortex-bound states as a function of lattice position.

along the vortex lines. These vortex-bound states are similar to the one-dimensional helical Majorana modes that exist at the edge of a fully gapped topological NCS in two dimensions. The numerical simulations of Fig. 2 are in excellent agreement with an analytical derivation of the vortex-bound states,,$[50$ cf. Appendix B and Ref. 39 .

The zero-energy vortex-bound states at $k_{z}=0$ are protected by a combination of $\pi$ rotation, time-reversal, and particle-hole symmetry; see Eq. (7a).51.53 Namely, we find that these zero energy modes are eigenstates of the chiral operator $\mathcal{S}=\mathcal{U}_{\mathrm{R}_{\pi}} \mathcal{U}_{T} \mathcal{U}_{P}$ and their stability is guaranteed by the conservation of chiral symmetry. That is, the doubly degenerate zero energy states at the vortex core are eigenstates of $\mathcal{S}$ with eigenvalue +1 , whereas the two zero-energy modes at the anti-vortex have eigenvalues -1 . Without breaking chiral symmetry, a zero-energy state in the vortex core with chirality eigenvalue +1 can only be removed together with a zero mode at the anti-vortex with chirality -1 . Hence, in the limit where vortex and anti-vortex cores are separated by a large distance, the zero-energy vortex-bound states are robust against any local perturbation that does not break chiral symmetry $\mathcal{S}$. In particular, the zero-energy vortex-bound states remain unperturbed by the chiral symmetric Zeeman field $h_{z} \sigma_{z} \otimes \sigma_{z}$.

By employing continuous deformations of the quasiparticle spectrum of Hamiltonian (1), one can show that the $\mathbb{Z}_{2}$ surface states and the helical vortex-bound states of the nodal NCS with $D_{4}$ point-group symmetry [phase III in Fig. 1](a)] originate from the nontrivial properties of the adjacent fully gapped phase of the $D_{4}$ NCS [phase I in Fig. 11.a)]. To be 
more specific, let us fix the momentum along the vortex line (e.g., to $k_{z}=0$ ) and consider adiabatic deformations connecting phase III to phase I that do not close the bulk gap at this particular momentum. During this deformation process, the two nodal rings shrink to nodal points at the north and south poles of the Fermi sphere and vanish, while the zero-energy vortex and surface states at $k_{z}=0$ remain unaffected. Moreover, the $\mathbb{Z}_{2}$ invariant $W_{\mathcal{A}}$ of the nodal phase III can be shown to be directly related to the $3 \mathrm{D}$ winding number $\nu_{3}$ of the fully gapped phase I (cf. Ref. 17). Hence, the zero-energy vortex and $\mathbb{Z}_{2}$ surface states of a nodal $D_{4}$ NCS are adiabatically connected to the vortex and surface states of a fully gapped topological NCS with $D_{4}$ point-group symmetry. A similar deformation process connecting phase III to phase II, on the other hand, does not exist, since upon crossing the transition line between phase III and phase I, the nodal rings approach each other and pair-annihilate. As a result, the zero-energy $\mathbb{Z}_{2}$ surface states and vortex-bound states disappear as one traverses the transition line.

\section{B. $C_{4 v}$ point-group NCSs}

Next we study surface and vortex-bound states of a nodal $C_{4 v}$ point-group NCS in phase III of Fig. 1 (b). The bulk quasiparticle spectrum in this nodal phase [Figs. 3. (a) and 3. b)] resembles the one of the $D_{4}$ NCS [Figs. 2,a) and 2,b)] and shows two nodal rings around the poles of the Fermi sphere. These line nodes have a nontrivial topological charge, which, as a consequence of the bulk-boundary correspondence, lead to the appearance of flat-band surface states. In addition, $C_{4 v}$ NCSs support helical arc surface states, that connect the projected nodal rings in the surface BZ [see Figs. 3 (c) and 3(d)]. These helical arc surface states are protected by a two-dimensional $\mathbb{Z}_{2}$ number, which is defined for each plane perpendicular to the (001) direction, (i.e., for planes with fixed $\left.k_{z}\right)^{\sqrt{23}}$, see Appendix C. The arc surface states of $C_{4 v}$ NCSs can be viewed as superconducting analogues of the Fermi arcs in time-revesal symmetric Weyl semimetals 54 , or alternatively as time-reversal invariant versions of the arc states in the A phase of superfluid ${ }^{3} \mathrm{He}^{57 / 58}$.

Due to the bulk-vortex correspondence 57 , vortex lines in $C_{4 v}$ NCSs support zero-energy bound states which are dispersionless along the vortex line. This is illustrated in Figs. 3.e) and 3 (f) for a pair of vortex and antivortex lines that are oriented along the $z$ axis. Just as the arc surface states, these flatband vortex-bound states connect the projected bulk nodes in $k_{z}$ momentum space. Following similar arguments as in Sec. IV A it can be shown that the zero-energy vortex-bound states of the $C_{4 v}$ NCSs for any fixed $k_{z}$ are protected by the chiral symmetry $\mathcal{S}=\mathcal{U}_{\mathrm{R}_{y}} \mathcal{U}_{T} \mathcal{U}_{P}$; see Eq. (7b). Using an adiabatic deformation of the Bogoliubov quasiparticle spectrum that does not close the bulk energy gap at the momenta $k_{z}$ in between the two projected nodal rings, we find that the flat-band vortex states and the arc surface states of the $C_{4 v}$ NCSs can be related to the vortex states and surface states of a time-reversal symmetric Weyl superconductor. That is, upon approaching the boundary of phase III in Fig. 11.b) where

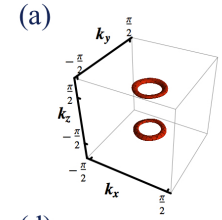

(d)

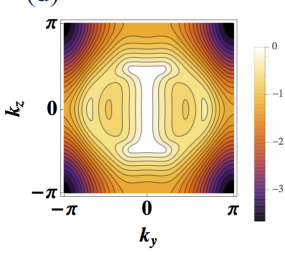

(e)
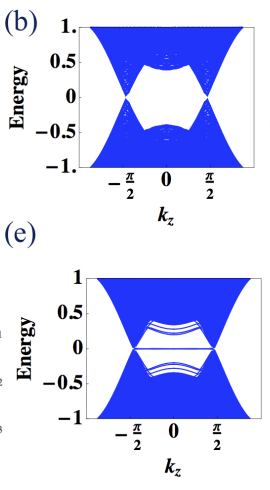

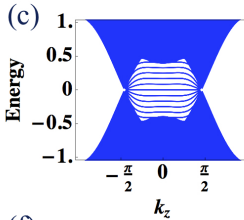

(f)
FIG. 3. (Color online). Same as Fig. 2 but for a $C_{4 v}$ point-group NCS with $a_{1}=1.0, \mu=-2.5$, and $\Delta_{s}=0.5$, corresponding to phase III in Fig. 1. b). (a) (b)

(c)

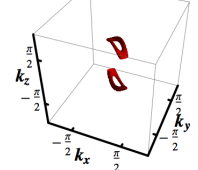

(e)

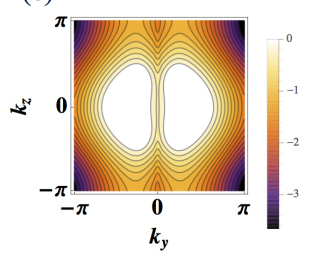

(c)

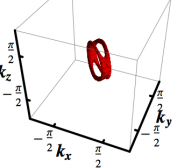

(f)

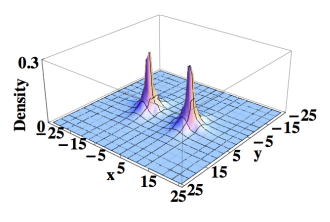

FIG. 4. (Color online). Bulk nodal structure, surface states, and finite-energy vortex-bound states for a $C_{2}$ point-group NCS with $a_{1}=a_{2}=1.0, a_{3}=0.3$, and $a_{4}=a_{5}=0.5$. Energies are measured in the unit of hopping. (a)-(d): Evolution of the bulk nodal structure as one moves along a path in the $\left(\mu, \Delta_{s}\right)$ phase diagram of Fig. 1 from phase III [panels (a) and (b) with $\left(\mu, \Delta_{s}\right)=(-2.5,0.5)$ and $(-2.9,0.5)$, respectively] to phase IV [panels (c) and (d) with $\left(\mu, \Delta_{s}\right)=(-3.1,0.5)$ and $(-3.1,1.5)$, respectively]. (e) and (f): Energy spectrum in the absence of vortices for (e) the highest negative-energy state and (f) all the states with $k_{y}=0$ of a $C_{2}$ NCS in a (100) slab geometry with the same parameters as in panel (d). (g) Energy spectrum in the presence of a vortex-antivortex pair oriented along the (110) direction as a function of momentum parallel to the vortex lines, $k_{\|}=\frac{1}{2}\left(k_{x}+k_{y}\right)$, with the same parameters as in panel (d). The subgap states are localized at the vortex cores.

$\Delta_{s}=0$ and $\mu>-3$, the nodal rings shrink to points at the north and south poles of the Fermi sphere and the $C_{4 v}$ NCSs turns into a time-reversal invariant Weyl superconductor, i.e., a time-reversal symmetric analog of the A phase of ${ }^{3} \mathrm{He}$.

\section{C. $C_{2}$ point-group NCSs}

Lastly, we examine the surface and vortex-bound states of NCSs with $C_{2}$ point-group symmetry. The phase diagram of 
$C_{2}$ NCSs as a function of spin-singlet pairing amplitude $\Delta_{s}$ and chemical potential $\mu$ displays two distinct nodal phases, which differ in the orientation of the nodal rings [Figs. 11.c), [1(d), and $4(a)-4(d)]$. In phase III the nodal rings are oriented along the (001) axis, while in phase IV they are centered about the (110) direction. As in the previous two cases, the topological characteristics of these nodal rings, which is described by the one-dimension winding number $\nu_{1}$, leads to the appearance of flat-band surface states. In addition, phase III supports $\mathbb{Z}_{2}$ Majorana surface states, whereas phase IV does not exhibit any additional surface states. This is exemplified in Figs. 4(e) and 4 (f), which show the energy spectrum at the (100) surface of a $C_{2}$ NCS in phase IV. Flat-band surface states appear within regions of the surface BZ that are bounded by the projected bulk nodal rings. But otherwise there exist no additional surface states in phase IV. Indeed, the energy spectrum along the $k_{z}=0$ line is fully gapped [Fig. 4(f)]. Using the same adiabatic deformations as before, we find that phase III can be connected to phase I, showing that the $\mathbb{Z}_{2}$ surface states of phase III originate from the topological properties of the fully gapped phase I. Phase IV, on the other hand, can be deformed into phase II by shrinking the nodal rings into points at opposite sides of the Fermi surface until they vanish, which corroborates our finding that there are no additional surface states in phase IV.

In contrast to NCSs with $D_{4}$ or $C_{4 v}$ point-group symmetry, NCSs with monoclinic point-group $C_{2}$ do not support any zero-energy vortex-bound states, neither in the fully gapped phases I and II nor in the nodal phases III and IV. This is in line with our finding that the chiral symmetry $\mathcal{S}$ (i.e., the combination of reflection ( $\pi$ rotation), particle-hole, and time-reversal symmetry), which is present for $D_{4}$ and $C_{4 v}$ NCSs but absent for $C_{2}$ NCSs, guarantees the the stability of the zero-energy vortex-bound states. The absence of zero-energy vortex states in $C_{2}$ point-group NCSs is demonstrated in Fig. $4 \mathrm{~g}$ ) for phase IV, which shows the energy spectrum for a vortex-antivortex pair oriented along the (110) axis, and also follows from an analytical argument,$[59]$ see Appendix $B$.

\section{Summary and discussion}

In summary, we have studied zero-energy vortex-bound states in 3D nodal and fully gapped NCSs. While vortex lines in NCSs with tetragonal point-group $D_{4}$ and $C_{4 v}$ support zero-energy vortex-bound states, $C_{2}$ point-group NCSs do not exhibit any Majorana vortex-bound states. We have found that the existence of Majorana vortex-bound states in nodal NCSs correlates with the appearance of Majorana cone and arc surface states. The zero-energy vortex states in $C_{4 v}\left(D_{4}\right) \mathrm{NCSs}$ are protected by a combination of reflection ( $\pi$ rotation), timereversal, and particle-hole symmetry, see Eq. (7). This is reminiscent of the zero modes at dislocation lines of bandtopological insulators which are stabilized by certain space group symmetries ${ }^{60}$.

Our findings have implications for experiments on 3D NCSs and on heterostructures, in which topological superconductivity is induced via the proximity effect of a conven- tional $s$-wave superconductor ${ }^{6162}$. Vortex-bound states can be directly observed in ordinary and spin-resolved scanning tunneling microscopy 36 . The helical Majorana vortex states of $D_{4}$ point-group NCSs can carry currents along the vortex lines, which could in principle be detected using thermal transport measurements $\mathrm{s}^{41}$. Moreover, the vortex-bound states are expected to be observable in terms of the cross-correlated responses between the orbital angular momentum $\mathbf{L}$ and the thermal polarization $\mathbf{P}_{E}$ of a 3D topological SC, which were recently discussed in Ref. 64. The so-called gravitomagnetoelectric polarizability of a 3D topological SC (i.e., the analog of the magnetoelectric polarizability of a 3D topological insulator) is given by

$$
\chi_{g}^{a b}=\frac{\partial L^{a}}{\partial E_{g}^{b}}=\frac{\partial P_{E}^{a}}{\partial \Omega^{b}}, \quad a, b=x, y, z,
$$

where $\Omega$ is the (external) angular velocity of the SC and $\mathbf{E}_{g}=-T^{-1} \boldsymbol{\nabla} T$ represents the temperature gradient. Note that the thermal polarization $\mathbf{P}_{E}$ is related to the distribution of the induced heat $Q$ via $\Delta Q=-\nabla \cdot \mathbf{P}_{E}$. According to Eq. (11) a thermal polarization (entropy polarization) $P_{E}^{a}$ can be generated by rotating the system with angular velocity $\Omega^{b}$. The presence of vortex lines leads to an additional contribution to the angular momentum and hence to an additional accumulation of entropy (heat) at the top and bottom surfaces of the 3D SC. Vortex-bound states, on the other hand, can carry a thermal current connecting top and bottom surfaces.

\section{Acknowledgments}

We thank J. C. Y. Teo, P. M. R. Brydon, and C. Timm for useful discussions. SR is supported by Alfred P. Sloan Research Fellowship (FG-BR2014-029).

\section{A. $q$ matrix}

A Hamiltonian $H=\sum_{\mathbf{k}} \Psi_{\mathbf{k}}^{\dagger} \mathcal{H}(\mathbf{k}) \Psi_{\mathbf{k}}$ which preserves chiral symmetry $S$ can be brought into block off-diagonal form,

$$
\tilde{\mathcal{H}}(\mathbf{k})=V \mathcal{H}(\mathbf{k}) V^{\dagger}=\left(\begin{array}{cc}
0 & D(\mathbf{k}) \\
D^{\dagger}(\mathbf{k}) & 0
\end{array}\right),
$$

where $V$ is a unitary transformation that diagonalizes the chiral symmetry operator $S$. In general, one can always deform a Hamiltonian into a flat-band Hamiltonian without altering its topological features. The flat-band Hamiltonian $Q(\mathbf{k})$ of Hamiltonian $\tilde{\mathcal{H}}(\mathbf{k})$ can be defined in terms of the spectral projector $P(\mathbf{k})$

$$
\begin{aligned}
Q(\mathbf{k}) & =\mathbb{I}_{4 N}-2 P(\mathbf{k}) \\
& =\mathbb{I}_{4 N}-2 \sum_{a=1}^{2 N}\left(\begin{array}{c}
\lambda_{a}^{-}(\mathbf{k}) \\
\mu_{a}^{-}(\mathbf{k})
\end{array}\right)\left(\left[\lambda_{a}^{-}(\mathbf{k})\right]^{\dagger}\left[\mu_{a}^{-}(\mathbf{k})\right]^{\dagger}\right),
\end{aligned}
$$


where $\left(\lambda_{a}^{-}(\mathbf{k}) \mu_{a}^{-}(\mathbf{k})\right)^{\mathrm{T}}$ are the negative-energy eigenfunctions of $\tilde{\mathcal{H}}(\mathbf{k})$, which are obtained from the eigenequation

$$
\left(\begin{array}{cc}
0 & D(\mathbf{k}) \\
D^{\dagger}(\mathbf{k}) & 0
\end{array}\right)\left(\begin{array}{c}
\lambda_{a}^{ \pm}(\mathbf{k}) \\
\mu_{a}^{ \pm}(\mathbf{k})
\end{array}\right)= \pm E_{a}(\mathbf{k})\left(\begin{array}{c}
\lambda_{a}^{ \pm}(\mathbf{k}) \\
\mu_{a}^{ \pm}(\mathbf{k})
\end{array}\right) .
$$

Here, $a=1, \ldots, 2 N$ denotes the combined band and spin index (we consider $N$ bands and two spin degrees of freedom). The eigenstates $\left(\lambda_{a}^{ \pm}(\mathbf{k}), \mu_{a}^{ \pm}(\mathbf{k})\right)^{\mathrm{T}}$ can be obtained from the eigenstates of $D(\mathbf{k}) D^{\dagger}(\mathbf{k})$ or $D^{\dagger}(\mathbf{k}) D(\mathbf{k})$,

$$
\begin{aligned}
& D(\mathbf{k}) D^{\dagger}(\mathbf{k}) \lambda_{a}(\mathbf{k})=E_{a}(\mathbf{k})^{2} \lambda_{a}(\mathbf{k}) \\
& D^{\dagger}(\mathbf{k}) D(\mathbf{k}) \mu_{a}(\mathbf{k})=E_{a}(\mathbf{k})^{2} \mu_{a}(\mathbf{k})
\end{aligned}
$$

where the eigenstates $\lambda_{a}(\mathbf{k})$ and $\mu_{a}(\mathbf{k})$ are normalized to be 1, i.e., $\lambda_{a}^{\dagger}(\mathbf{k}) \lambda_{a}(\mathbf{k})=\mu_{a}^{\dagger}(\mathbf{k}) \mu_{a}(\mathbf{k})=1$. With this, we find that the eigenstates of $\tilde{\mathcal{H}}(\mathbf{k})$ are $\left(\lambda_{a}^{ \pm}(\mathbf{k}), \mu_{a}^{ \pm}(\mathbf{k})\right)^{\mathrm{T}}=$ $\frac{1}{\sqrt{2}}\left(\lambda_{a}(\mathbf{k}), \pm \mu_{a}(\mathbf{k})\right)^{\mathrm{T}}$. Hence the flat-band Hamiltonian $Q(\mathbf{k})$ defined from the spectral project $P(\mathbf{k})$ holds this off- diagonal form,

$$
Q(\mathbf{k})=\mathbb{I}_{4 N}-2 P(\mathbf{k})=\left(\begin{array}{cc}
0 & q(\mathbf{k}) \\
q^{\dagger}(\mathbf{k}) & 0
\end{array}\right),
$$

where $q(\mathbf{k})=\sum_{a} \lambda_{a}(\mathbf{k}) \mu_{a}^{\dagger}(\mathbf{k})$, for more details; see Refs. 19 and 22.

\section{B. Continuum BdG equations}

The continuum BdG equation can be expressed as

$$
\mathcal{H} \psi=\left(\begin{array}{cc}
h & \Delta \\
\Delta^{\dagger} & -h^{*}
\end{array}\right) \psi=E \psi
$$

where $h=\left(-\frac{\nabla^{2}}{2 m}-\mu\right) \mathbb{I}_{2 \times 2}+\alpha \mathbf{l}(\mathbf{k}) \cdot \boldsymbol{\sigma}$, with $m$ the effective mass, $\mu$ the chemical potential, and $\alpha \mathbf{l}(\mathbf{k}) \cdot \boldsymbol{\sigma}$ the Rashba-type SOC with strength $\alpha$. The pairing term has the form $\Delta=$ $\left(\Delta_{s}+\frac{1}{2} \boldsymbol{\nabla} \cdot \mathbf{D}+\mathbf{D} \cdot \boldsymbol{\nabla}\right)\left(i \sigma_{2}\right)$, where $\Delta_{s}$ is the singlet pairing amplitude and $\mathbf{D}=-i \nabla_{\mathbf{k}}\left(\Delta_{p} \mathbf{l}(\mathbf{k}) \cdot \boldsymbol{\sigma}\right)$ presents the triplet pairing. Here, $\boldsymbol{\sigma}=\left(\sigma_{1}, \sigma_{2}, \sigma_{3}\right)$ is the vector of Pauli matrices. Without loss of generality, we consider the spin-orbit coupling vector $\mathbf{l}(\mathbf{k})=\left(a_{1} k_{x}+a_{4} k_{y}, a_{1} k_{y}+a_{4} k_{x}, a_{3} k_{z}\right)$. A vortex line along the $z$ direction can be introduced by adding a phase on both singlet and triplet pairing amplitudes, $\Delta_{s} \rightarrow e^{i \theta} \Delta_{s}$ and $\Delta_{t} \rightarrow e^{i \theta} \Delta_{t}$. In the cylindrical coordinate, the normal-state Hamiltonian and the pairing term are

$$
\begin{aligned}
h= & \left(-\frac{1}{2 m}\left(\partial_{r}^{2}+\frac{1}{r} \partial_{r}+\frac{1}{r^{2}} \partial_{\theta}^{2}+\partial_{z}^{2}\right)-\mu\right) \mathbb{I}_{2 \times 2} \\
& +\alpha\left(\begin{array}{cc}
a_{3}\left(-i \partial_{z}\right) & -i a_{1} e^{-i \theta}\left(\partial_{r}-\frac{i}{r} \partial_{\theta}\right)-i a_{4} e^{i \theta}\left(-i \partial_{r}+\frac{1}{r} \partial_{\theta}\right) \\
-i a_{1} e^{i \theta}\left(\partial_{r}+\frac{i}{r} \partial_{\theta}\right)-i a_{4} e^{-i \theta}\left(i \partial_{r}+\frac{1}{r} \partial_{\theta}\right) & a_{3}\left(i \partial_{z}\right)
\end{array}\right) \\
\Delta= & \Delta_{s} e^{i \theta}\left(i \sigma_{2}\right) \\
& -i \Delta_{t}\left(\begin{array}{cc}
a_{1}\left(-\partial_{r}+i \frac{1}{r} \partial_{\theta}-\frac{1}{2 r}\right)+i e^{2 i \theta} a_{4}\left(\partial_{r}+i \frac{1}{r} \partial_{\theta}-\frac{1}{2 r}\right) & a_{3} \partial_{z} \\
a_{3} \partial_{z} & e^{2 i \theta} a_{1}\left(\partial_{r}+i \frac{1}{r} \partial_{\theta}-\frac{1}{2 r}\right)+i a_{4}\left(\partial_{r}-i \frac{1}{r} \partial_{\theta}+\frac{1}{2 r}\right)
\end{array}\right) .
\end{aligned}
$$

A general solution of this continuum BdG equation is $\psi(r, \theta, z)=e^{i k_{z} z}\left[\tilde{u}_{\uparrow}(r) e^{i m_{1} \theta}, \tilde{u}_{\downarrow}(r) e^{i m_{2} \theta}, \tilde{v}_{\uparrow}(r) e^{i n_{1} \theta}, \tilde{v}_{\downarrow}(r) e^{i n_{2} \theta}\right]^{\mathrm{T}}$. The radial part of a localized solution must be of the form $f(r) \sim e^{-\kappa r}$ with $\operatorname{Re}[\kappa]>0$. In the asymptotic limit $(1 / r \rightarrow 0)$, we can neglect all $\frac{1}{r}$ and $\frac{1}{r^{2}}$ terms. We find for $\left(m_{1}, m_{2}, n_{1}, n_{2}\right)=(0,1,0,-1)$ and $a_{4}=0$, that the continuum BdG equation has a localized zero-energy solution for (i) $k_{z}=0$ or (ii) $a_{3}=0$, that satisfies

$$
\left(\begin{array}{cccc}
-\frac{1}{2 m} \kappa^{2}-\mu & i \alpha a_{1} \kappa e^{-i \theta} & -i \Delta_{t} a_{1} \kappa & \Delta_{s} e^{i \theta} \\
i \alpha a_{1} \kappa e^{i \theta} & -\frac{1}{2 m} \kappa^{2}-\mu & -\Delta_{s} e^{i \theta} & i \Delta_{t} a_{1} \kappa e^{2 i \theta} \\
i \Delta_{t} a_{1} \kappa & -\Delta_{s} e^{-i \theta} & \frac{1}{2 m} \kappa^{2}+\mu & -i \alpha a_{1} \kappa e^{i \theta} \\
\Delta_{s} e^{-i \theta} & -i \Delta_{t} a_{1} \kappa e^{-2 i \theta} & -i \alpha a_{1} \kappa e^{-i \theta} & \frac{1}{2 m} \kappa^{2}+\mu
\end{array}\right)\left(\begin{array}{c}
\tilde{u}_{\uparrow}(r) \\
\tilde{u}_{\downarrow}(r) e^{i \theta} \\
\tilde{v}_{\uparrow}(r) \\
\tilde{v}_{\downarrow}(r) e^{-i \theta}
\end{array}\right)=0
$$


Note that this situation corresponds to a zero-energy bound-state solution at $k_{z}=0$ for the $D_{4}$ point-group NCS. In addition, the decay length $\kappa$ is determined by solving the roots of the following determinant

$$
\operatorname{Det}\left(\begin{array}{cccc}
-\frac{1}{2 m} \kappa^{2}-\mu & i \alpha a_{1} \kappa & -i \Delta_{t} a_{1} \kappa & \Delta_{s} \\
i \alpha a_{1} \kappa & -\frac{1}{2 m} \kappa^{2}-\mu & -\Delta_{s} & i \Delta_{t} a_{1} \kappa \\
i \Delta_{t} a_{1} \kappa & -\Delta_{s} & \frac{1}{2 m} \kappa^{2}+\mu & -i \alpha a_{1} \kappa \\
\Delta_{s} & -i \Delta_{t} a_{1} \kappa & -i \alpha a_{1} \kappa & \frac{1}{2 m} \kappa^{2}+\mu
\end{array}\right)=0
$$

There are only two decaying solutions $(\operatorname{Re}[\kappa]>0)$

$$
\kappa_{ \pm}=\sqrt{-2 a_{1}^{2} m^{2}\left(\alpha^{2}+\Delta_{t}^{2}\right)-2 m \mu \pm 2 \sqrt{\left[a_{1}^{2} m^{2}\left(\alpha^{2}+\Delta_{t}^{2}\right)+m \mu\right]^{2}-m^{2}\left(\Delta_{s}^{2}+\mu^{2}\right)}}
$$

with the condition

$$
-a_{1}^{2} m^{2}\left(\alpha^{2}+\Delta_{t}^{2}\right)-m \mu-\operatorname{Re}\left[\sqrt{\left[a_{1}^{2} m^{2}\left(\alpha^{2}+\Delta_{t}^{2}\right)+m \mu\right]^{2}-m^{2}\left(\Delta_{s}^{2}+\mu^{2}\right)}\right]>0
$$

(a)

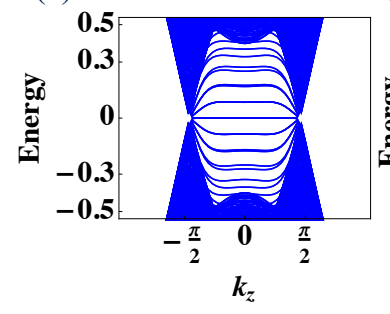

(b)

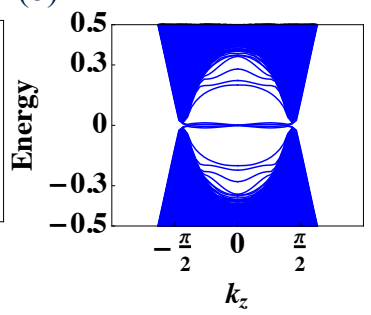

FIG. 5. (Color online). Energy spectrum as a function of $k_{z}$ of a $C_{4 v}$ NCS with $\left(a_{1}, a_{2}\right)=(1.0,2.0), \mu=-2.5$, and $\Delta_{s}=0.5$. (a) Without vortices and OBC along the $x$ axis but PBCs in the other two directions. (b) With a pair of vortex-antivortex lines oriented along the $z$ axis and PBCs in all three directions.

\section{Higher-order SOC in NCSs with $C_{4 v}$ point-group symmetry}

For the $C_{4 v}$ NCS with $\mathbf{l}(\mathbf{k})$ given by Eq. (3b) (i.e., only the lowest order SOC term) one finds that the BdG Hamiltonian $\mathcal{H}(\mathbf{k})$ is an even function of $k_{z}$. Hence, an effective two-dimensional layer with fixed $k_{z}$ satisfies timereversal and particle-hole symmetries, $U_{T}^{-1} \mathcal{H}\left(k_{x}, k_{y}\right) U_{T}=$ $\mathcal{H}^{*}\left(-k_{x},-k_{y}\right)$ and $U_{P}^{-1} \mathcal{H}\left(k_{x}, k_{y}\right) U_{P}=-\mathcal{H}^{*}\left(-k_{x},-k_{y}\right)$. It turns out that each layer with fixed $k_{z}$ belongs to class DIII and the two-dimensional $\mathbb{Z}_{2}$ topological invariant $(10)$ can be computed. For $k_{z}$ within the two nodal rings, we find that the $\mathbb{Z}_{2}$ number takes on a nontrivial value, which leads to the appearance of helical arc surface states.

It is interesting to ask whether this reasoning remains valid upon inclusion of higher-order terms in the spin-orbit interaction. Up to second order the SOC vector $\mathbf{l}(\mathbf{k})$ for the $C_{4 v}$ point-group is given by

$$
\begin{aligned}
\mathbf{l}(\mathbf{k})= & a_{1}\left[\sin k_{y} \hat{\mathbf{e}}_{1}-\sin k_{x} \hat{\mathbf{e}}_{2}\right] \\
& +a_{2} \sin k_{x} \sin k_{y} \sin k_{z}\left(\cos k_{x}-\cos k_{y}\right) \hat{\mathbf{e}}_{3} .
\end{aligned}
$$

We observe that the second-order term is an odd function of $k_{z}$, and hence two-dimensional layers with fixed $k_{z}$ are no longer symmetric under TRS and PHS. Our numerics shows that upon inclusion of the second-order term with $a_{2} \neq 0$, the flat-band vortex-bound states become dispersive [Fig. 5(b)]. However, the arc surface states remain unaffected [Fig. 5(a)]. It turns out that the arc surface states on the (010) and (100) faces are protected by a reflection symmetry which leaves the surface plane invariant. The Hamiltonian in a (010) slab geometry is invariant under the following reflection symmetry:

$$
\mathcal{U}_{\mathrm{R}_{x}}^{\dagger} \tilde{\mathcal{H}}\left(y, y^{\prime} ; k_{x}, k_{z}\right) \mathcal{U}_{\mathrm{R}_{x}}=\tilde{\mathcal{H}}\left(y, y^{\prime} ;-k_{x}, k_{z}\right),
$$

with $\mathcal{U}_{\mathrm{R}_{x}}=\delta_{y, y} \otimes\left[\sigma_{3} \otimes \sigma_{1}\right]$, where $\delta_{y, y}$ acts on the real-space basis and $\sigma_{3} \otimes \sigma_{1}$ acts on the momentum-space Nambu basis. The Hamiltonian in a (100) geometry, on the other hand, is invariant under the following mirror symmetry:

$$
\mathcal{U}_{\mathrm{R}_{y}}^{\dagger} \tilde{\mathcal{H}}\left(x, x^{\prime} ; k_{y}, k_{z}\right) \mathcal{U}_{\mathrm{R}_{y}}=\tilde{\mathcal{H}}\left(x, x^{\prime} ;-k_{y}, k_{z}\right),
$$

with $\mathcal{U}_{\mathrm{R}_{y}}=\delta_{x, x} \otimes\left[\mathbb{I}_{2 \times 2} \otimes \sigma_{2}\right]$, where $\delta_{x, x}$ acts on the real-space basis and $\mathbb{I}_{2 \times 2} \otimes \sigma_{2}$ acts on the momentum-space Nambu basis. Here, $\tilde{\mathcal{H}}\left(y, y^{\prime} ; k_{x}, k_{z}\right)$ and $\tilde{\mathcal{H}}\left(x, x^{\prime} ; k_{y}, k_{z}\right)$ represent the tight-binding Hamiltonians of the $C_{4 v}$ NCS in a (010) and (100) slab geometry, respectively. Note that the arc surface states on the (010) face [(100) face] are left invariant by the reflection symmetry $\mathrm{R}_{x}\left[\mathrm{R}_{y}\right]$ and that both reflection operators $\mathcal{U}_{\mathrm{R}_{x}}$ and $\mathcal{U}_{\mathrm{R}_{y}}$ have eigenvalues +1 and -1 . Since the reflection operator $\mathcal{U}_{\mathrm{R}_{x}}\left[\mathcal{U}_{\mathrm{R}_{y}}\right]$ commutes with $\tilde{\mathcal{H}}\left(y, y^{\prime} ; k_{x}=0, k_{z}\right)\left[\tilde{\mathcal{H}}\left(x, x^{\prime} ; k_{y}=0, k_{z}\right)\right]$, the eigenfunctions of $\tilde{\mathcal{H}}\left(y, y^{\prime} ; k_{x}=0, k_{z}\right)\left[\tilde{\mathcal{H}}\left(x, x^{\prime} ; k_{y}=0, k_{z}\right)\right]$ are simultaneous eigenstates of $\mathcal{U}_{\mathrm{R}_{x}}\left[\mathcal{U}_{\mathrm{R}_{y}}\right]$ with eigenvalues +1 or -1 . We have checked that the two helical zero-energy surface states belong to different eigenspaces of $\mathcal{U}_{\mathrm{R}_{x}}\left[\mathcal{U}_{\mathrm{R}_{y}}\right]$. Thus these zero-energy states cannot hybridize and are protected by these reflection symmetries. 
${ }^{1}$ M. Z. Hasan and C. L. Kane, Rev. Mod. Phys. 82, 3045 (2010).

2 X.-L. Qi and S.-C. Zhang, Rev. Mod. Phys. 83, 1057 (2011).

3 S. Ryu, A. P. Schnyder, A. Furusaki, and A. W. W. Ludwig, New J. Phys., 12, 065010 (2010).

${ }^{4}$ C. W. J. Beenakker, Annu. Rev. Condens. Matter Phys. 2, 55 (2011).

5 J. Alicea, Rep. Prog. Phys. 75, 6501(2012).

${ }^{6}$ V. Mourik, K. Zuo, S. M. Frolov, S. R. Plissard, E. P. A. M. Bakkers, and L. P. Kouwenhoven, Science 336, 6084 (2012).

7 A. Das, Y. Ronen, Y. Most, Y. Oreg, M. Heiblum, and H. Shtrikman, Nat. Phys. 8, 887 (2012).

${ }^{8}$ M. T. Deng, C. L. Yu, G. Y. Huang, M. Larsson, P. Caroff, and H. Q. Xu, Nano. Lett. 12, 6414 (2012).

9 E. J. H. Lee, X. Jiang, M. Houzet, R. Aguado, C. M. Lieber, and S. De Franceschi, Nat. Nanotechnol. 9, 79 (2014).

10 J. D. Sau, R. M. Lutchyn, S. Tewari, and S. Das Sarma, Phys. Rev. Lett. 104, 040502 (2010).

11 R. M. Lutchyn, J. D. Sau and S. Das Sarma Phys. Rev. Lett. 105, 077001(2010).

12 Y. Oreg, G. Refael, and F. von Oppen, Phys. Rev. Lett. 105, 177002 (2010).

13 L. Fu and C. L. Kane, Phys. Rev. Lett. 100, 096407 (2008).

${ }^{14}$ E. Bauer and M. Sigrist, Non-Centrosymmetric Superconductors: Introduction and Overview, Lect. Notes Phys. 847, 1-357 (2012).

15 M. Sato, Phys. Rev. B 73214502 (2006).

16 Y. Tanaka, Y. Mizuno, T. Yokoyama, K. Yada, and M. Sato, Phys. Rev. Lett. 105097002 (2010).

17 M. Sato, and S. Fujimoto, Phys. Rev. Lett. 105217001 (2010).

18 B. Béri, Phys. Rev. B 81134515 (2010).

19 A. P. Schnyder and S. Ryu, Phys. Rev. B 84060504 (2011).

${ }^{20}$ K. Yada, M. Sato, Y. Tanaka, and T. Yokoyama, Phys. Rev. B 83 064505 (2011).

${ }^{21}$ P. M. R. Brydon, A. P. Schnyder, and C. Timm, Phys. Rev. B 84 020501 (2011).

22 S. Matsuura, P. -Y. Chang, A. P. Schnyder, S. Ryu, New J. Phys. 15065001 (2013).

23 A. P. Schnyder, P. M. R. Brydon, and C. Timm, Phys. Rev. B 85 024522 (2012).

${ }^{24}$ M. Sato and S. Fujimoto, Phys. Rev. B 79094504 (2009).

25 E. Bauer, G. Hilscher, H. Michor, Ch. Paul, E. W. Scheidt, A. Gribanov, Yu. Seropegin, H. Noe?1, M. Sigrist, and P. Rogl, Phys. Rev. Lett. 92027003 (2004).

${ }^{26}$ K. Izawa, Y. Kasahara, Y. Matsuda, K. Behnia, T. Yasuda, R. Settai, and Y. Onuki, Phys. Rev. Lett. 94, 197002 (2005).

${ }^{27}$ I. Bonalde, R. L. Robeiro, W. Brämer-Escamilla, C. Rojas, E. Bauer, A. Prokofiev, Y. Haga, T. Yasuda, and Y. Ōnuki, New. J. Phys. 11, 055054 (2009).

${ }^{28}$ H. Mukuda, T. Fujii, T. Ohara, A. Harada, M. Yashima, Y. Kitaoka, Y. Okuda, R. Settai, and Y. Onuki, Phys. Rev. Lett. 100, 107003 (2008)

${ }^{29}$ N. Kimura, K. Ito, K. Saitoh, Y. Umeda, H. Aoki, and T. Terashima, Phys. Rev. Lett. 95, 247004 (2005).

30 J. Chen, M. B. Salamon, S. Akutagawa, J. Akimitsu, J. Singleton, J. L. Zhang, L. Jiao, and H. Q. Yuan, Phys. Rev. B 83, 144529 (2011).

${ }^{31}$ H. Q. Yuan, D. F. Agterberg, N. Hayashi, P. Badica, D. Vandervelde, K. Togano, M. Sigrist, and M. B. Salamon, Phys. Rev. Lett. 97, 017006 (2006).

${ }^{32}$ M. Nishiyama, Y. Inada, and G.-Q. Zheng, Phys. Rev. Lett. 98, 047002 (2007)

${ }^{33}$ G. Eguchi, D. C. Peets, M. Kriener, S. Yonezawa, G. Bao, S.
Harada, Y. Inada, G.-q. Zheng, and Y. Maeno, Phys. Rev. B 87, 161203(R) (2013).

${ }^{34}$ M. Mondal, B. Joshi, S. Kumar, A. Kamlapure, S. C. Ganguli, A. Thamizhavel, S. S. Mandal, S. Ramakrishnan, and P. Raychaudhuri, Phys. Rev. B 86, 094520 (2012).

35 M. Sato, Y. Tanaka, K. Yada, and T. Yokoyama, Phys. Rev. B 83 224511 (2011).

36 J. S. Hofmann, R. Queiroz, and A. P. Schnyder, Phys. Rev. B 88, 134505 (2013)

37 P. M. R. Brydon, C. Timm, A. P. Schnyder, New J. Phys. 15, 045019 (2013).

38 A. P. Schnyder, Carsten Timm, and P. M. R. Brydon, Phys. Rev. Lett. 111077001 (2013).

39 C. -K. Lu and S. Yip, Phys. Rev. B 78132502 (2008).

${ }^{40}$ S. Fujimoto, Phys. Rev. B 77 220501(R) (2008).

${ }^{41}$ M. K. Kashyap and D. F. Agterberg, Phys. Rev. B 88104515 (2013).

${ }^{42}$ For previous studies on stable Majorana vortex-bound states in fully gapped 2D NCSs, in 3D SCs with Rashba-spin orbit coupling, and NCSs with cubic crystal symmetry $O$, see Refs. 24, 39. 41.

43 This is in some sense expected since helical modes are unstable against TRS breaking perturbation, and here vortices break TRS.

${ }^{44}$ X. Wan, A. M. Turner, A. Vishwanath, and S. Y. Savrasov, Phys. Rev. B 83, 205101 (2011).

45 A. A. Burkov and Leon Balents, Phys. Rev. Lett. 107, 127205 (2011).

46 G. Xu, H. Weng, Z. Wang, X. Dai, and Z. Fang, Phys. Rev. Lett. 107, 186806 (2011).

47 P. A. Frigeri, D. F. Agterberg, A. Koga, and M. Sigrist, Phys. Rev. Lett. 92, 097001 (2004).

48 K.V. Samokhin, Annals of Physics 3242385 (2009).

49 The gap closes when $0=|B-A| \mathbf{l}||$ with $B=\varepsilon(\mathbf{k})+i \Delta_{s}$ and $A=\alpha+i \Delta_{t}$. The gapless points are the intersections between hypersurfaces that are characterized by these two equations: $t\left(\cos k_{x}+\cos k_{y}+\cos k_{z}\right)=\mu+\alpha \Delta_{s} / \Delta_{t}$ and $|\mathbf{l}|=\Delta_{s} / \Delta_{t}$.

${ }^{50}$ Following Ref. 39, we consider the normal state band structure $h=\left(-\frac{\nabla^{2}}{2 m}-\mu\right) \mathbb{I}_{2 \times 2}+\alpha \mathbf{l}(\mathbf{k}) \cdot \boldsymbol{\sigma}$ and the pairing term $\Delta=\left(\Delta_{s}+\right.$ $\left.\frac{1}{2} \boldsymbol{\nabla} \cdot \mathbf{D}+\mathbf{D} \cdot \boldsymbol{\nabla}\right)\left(i \sigma_{2}\right)$, where $m$ is the effective mass and $\mathbf{D}=$ $-i \nabla_{\mathbf{k}}\left(\Delta_{p} \mathbf{l}(\mathbf{k}) \cdot \boldsymbol{\sigma}\right)$. We can introduce a vortex line along the $z$ axis localized at the origin by adding a phase on gap functions for both singlet and triplet pairings. In the continuum model, we need to linearize $\mathbf{l}(\mathbf{k})=\left(a_{1} k_{x}, a_{1} k_{y}, a_{3} k_{z}\right)$. In addition, we consider the asymptotic limit $(1 / r \rightarrow 0)$ that we can neglect all $\frac{1}{r}$ and $\frac{1}{r^{2}}$ terms in the continuum BdG equation, where $r$ is the radial direction in the cylindrical coordinate. As the result, there is a localized zero energy solution that decays as a function of $r$.

51 C. Fang, M. J. Gilbert, and B. A. Bernevig, Phys. Rev. Lett. 112, 106401 (2014).

52 T. Mizushima, M. Sato, and K. Machida, Phys. Rev. Lett. 109, 165301 (2012).

53 K. Shiozaki and M. Sato, Phys. Rev. B 90, 165114 (2014).

${ }^{54}$ The Fermi arcs discussed in Refs. $44-46$ are protected by a $\mathbb{Z}$ number. In our case, due to time-reversal symmetry, the helical arc surface states are protected by a $\mathbb{Z}_{2}$ number.

55 T. Ojanen, Phys. Rev. B 87, 245112 (2013).

56 T. Morimoto and A. Furusaki, Phys. Rev. B 89, 235127 (2014).

57 G. E. Volovik, JETP Lett. 9366 (2011).

${ }^{58}$ G. E. Volovik, in The Universe in a Helium Droplet, The International Series of Monographs on Physics Vol. 117 (Oxford Univer- 
sity Press, New York, 2003); G. E. Volovik, in Exotic Properties of Superfluid $3 \mathrm{He}$, Series I N Modern Condensed Matter Physics Vol. 1 (World Scientific, Singapore, 1992).

${ }^{59}$ For the $C_{2}$ point-group NCS with $a_{4} \neq 0$ and $a_{5} \neq 0$, the variables $r$ and $\theta$ are not separable in the continuum BdG equation given in the Appendix $B$, which may imply that there are no zeroenergy solutions localized at the vortex core.

${ }^{60}$ R.-J. Slager, A. Mesaros, V. Juricic, and J. Zaanen, arXiv:1401.4044.
${ }^{61}$ S. Sasaki, K. Segawa, and Y. Ando, arXiv:1404.1707.

${ }^{62}$ F. Zhang, C. L. Kane, and E. J. Mele, Phys. Rev. Lett. 111, 056402 (2013).

63 Z. Sun, M. Enayat, A. Maldonado, C. Lithgow, E. Yelland, D. C. Peets, A. Yaresko, A. P. Schnyder, and P. Wahl, arXiv:1407.5667.

${ }^{64}$ K. Nomura, S. Ryu, A. Furusaki, and N. Nagaosa, Phys. Rev. Lett. 108, 026802 (2012). 